\title{
Diffusive sampling of methylene chloride with solid phase microextraction
}

\author{
Cheng-Yao Chen ${ }^{\text {a }}$, Chunming Hsiech ${ }^{\text {a }}$, Jia-Ming Lin ${ }^{\text {b,* }}$ \\ ${ }^{a}$ Institute of Occupational Safety and Health, Council of Labor Affairs, Executive Yuan, Taipei, Taiwan \\ ${ }^{\mathrm{b}}$ Institute of Environmental Health, College of Public Health, National Taiwan University, \\ No.17 Xu-Zhou Road, Taipei 10020, Taiwan
}

Received 10 January 2006; received in revised form 5 October 2006; accepted 11 October 2006 Available online 2 November 2006

\begin{abstract}
This study examined the characteristics of a solid phase microextraction (SPME) assembly as a passive sampler to determine the short-term exposure level (STEL) of methylene chloride. Two types of SPME fibers and six sampling-related factors were chosen and nested in an $L_{18}$ Taguchi's orthogonal array. Samples were thermally desorpted and analyzed by gas chromatograph equipped with an electron capture detector (GC/ECD). The use of 85- $\mu \mathrm{m}$ Carboxen/polydimethylsiloxane (Car/PDMS) fibers resulted in greater adsorbed mass, which was highly correlated with the product of concentration and sampling time $(r>0.99, p<0.0001)$, than $85-\mu \mathrm{m}$ polyacrylate fibers. The sampling rate (SR) of the $85-\mu \mathrm{m}$ Carboxen/polydimethylsiloxane fibers was not significantly affected by variations in relative humidity (0-80\%) and coexistent toluene (none to $100 \mathrm{ppm})$. Variance of sampling rate was predominantly attributed to the diffusive path length (86.4\%) and sampling time (5.7\%). With diffusive paths of 3,10 and $15 \mathrm{~mm}$, the sampling rates of $85-\mu \mathrm{m}$ Carboxen/polydimethylsiloxane fibers for methylene chloride were $1.4 \times 10^{-2}, 7.7 \times 10^{-3}$ and $5.1 \times 10^{-3} \mathrm{~mL} \mathrm{~min}^{-1}$, respectively. The measured sampling rates were greater than the theoretical values, and decreased with increment of sampling time until they came to constant.
\end{abstract}

(C) 2006 Elsevier B.V. All rights reserved.

Keywords: Methylene chloride; Solid phase microextraction; Diffusive sampling

\section{Introduction}

Methylene chloride is a solvent used in various industrial processes such as paint stripping, metal surface cleaning and degreasing. Inhalation and skin contact are the major routes of exposure [1]. Fatal incidence has been reported due to the biotransformation of methylene chloride into carbon monoxide in vivo [2]. Because of its carcinogenicity, methylene chloride is listed by the International Agency for Research on Cancer (IARC) as a possible human carcinogen (Group 2B). While the US National Institute for Occupational Safety and Health (NIOSH) recommends that the exposure level be as feasible low as possible, the American Conference for Governmental Industrial Hygienists (ACGIH) recommends $50 \mathrm{ppm}$ as the threshold limit value $\left(\mathrm{TLV}^{\circledR}\right)$ for minimizing the potential elevation of

\footnotetext{
* Corresponding author. Tel.: +886 2 33228103; fax: +886 223918996

E-mail address: jmlin@ha.mc.ntu.edu.tw (J.-M. Lin).
}

carboxyhemoglobin and the depression of the central nervous system [3].

Inhalation exposure in a risk group is usually measured by an active charcoal sampling tube coupled with GC/FID, gas chromatograph equipped with an electron capture detector (GC/ECD) or GC/MS for analysis [4-8]. These integrated methods not only take time for sampling as well as sample preparation, but also consume solvent in the process of sample extraction. Solid phase microextraction (SPME) provides significant advantages over the traditional methods. It offers solvent-free operation, convenient coupling with field analytical instruments, good accuracy, precision, cost effectiveness and simplicity of operation and calibration [9].

Diffusive sampling techniques using SPME have been reported for gaseous chemicals such as hydrocarbons, ethylene oxide, and $n$-valeraldehyde, etc. based on Fick's first law [10-12]. According to the law, the mass loading of a diffusive sampler is proportionally related to the concentration $(C)$ of the chemical, the exposure time and the sampling rate (SR). 
The sampling rate is a product of the diffusion coefficient $(D)$ of a chemical and the ratio of the cross-sectional area of the diffusion path over the path length for diffusion of that sampler. The sampling rate of a diffusive sampler for a compound can be experimentally determined or calculated as the diffusion coefficient is estimated by Gilliland's approximation [13]. Thus, absolute temperature, $T$, is a factor affecting the performance of a diffusive sampler not only because the $D$ is proportional to $T^{1.5}$ and inversely proportional to pressure $(P)$, but also because the $C$ is proportional to $P$ and inversely proportional to $T$. As a result, the mass loading of a diffusive sampler is proportional to $T^{0.5}$.

In using an SPME assembly as a diffusive sampler, the collection medium is the coating material on a fused-silica fiber. There are two types of SPME fibers available. A critical part of the fiber selection process is determining whether to use an adsorbent type fiber or an absorbent type fiber. The affinity between the sorbent and the sorbate is one of the considerations when choosing an appropriate fiber. Typically, the chemical nature of a target analyte determines the type of fiber used [14]. In addition, the atmospheric conditions, especially relative humidity and coexistent compound, might create competitive problems of adsorption sites of a collection medium.

This paper was intended to characterize the performance of a SPME device as a diffusive sampler for methylene chloride based on the experimental trials designed with Taguchi's orthogonal array.

\section{Experimental}

\subsection{Design and statistical analyses}

As mentioned above, the performance of loading a chemical on a SPME fiber would depend on the configuration of a sam-
Table 1

Designation of factors and levels for each factor in experimental design by Taguchi's orthogonal array

\begin{tabular}{llllc}
\hline Factor & Variable & Level 1 & Level 2 & Level 3 \\
\hline A & Sorbent type & Car/PDMS & polyacrylate & - \\
B & Concentration $(\mathrm{ppm})$ & 25 & 50 & 100 \\
C & Temperature $\left({ }^{\circ} \mathrm{C}\right)$ & 15 & 25 & 30 \\
D & RH $(\%)$ & dry air & 50 & 80 \\
E & Interference, toluene $(\mathrm{ppm})$ & 0 & 50 & 100 \\
F & Path length $(\mathrm{mm})$ & 3 & 10 & 15 \\
$\mathrm{G}$ & Sampling time $(\mathrm{min})$ & 5 & 15 & 30 \\
$\mathrm{H}$ & (Not used) & - & - & - \\
\hline
\end{tabular}

pling device, the type of collection medium, the concentration of a chemical, the sampling time and the atmospheric conditions involving in relative humidity, ambient temperature and coexistent compounds. Thus, seven factors shown in Table 1 were chosen for the experimental design. A Taguchi's orthogonal array $L_{18}\left(2^{1} \times 3^{7}\right)[15]$ was used for this seven-factor experiment as shown in Table 2. $L$ and subscript 18 denote the Latin square and number of the experimental runs, respectively. Factor A had only two levels, while the others had three levels. The dual levels of factor A were coded as 1 and 2. The three levels of the other factors were coded as 1, 2 and 3. A run involved the corresponding combination of levels assigned to the seven factors.

The levels of each factor were determined according to the scenarios of industrially hygienic sampling that the SPME sampler would meet. The chosen polyacrylate fibers had the same $85-\mu \mathrm{m}$ thickness as the Carboxen/polydimethylsiloxane (Car/PDMS) fibers, but with different properties. Polyacrylate fiber is a polar absorbent while Car/PDMS fiber is a bipolar adsorbent. The concentration of methylene chloride was set for 25,50 and $100 \mathrm{ppm}$ to reflect to the threshold limit values of methylene chloride, $50 \mathrm{ppm}$, as $0.5,1$, and $2 \mathrm{TLV}^{\circledR}$ by ACGIH.

Table 2

Experimental trials and results of the $L_{18}$ orthogonal array design

\begin{tabular}{|c|c|c|c|c|c|c|c|c|c|c|}
\hline \multicolumn{9}{|c|}{$L_{18}$ orthogonal array design } & \multicolumn{2}{|l|}{ Experimental results } \\
\hline Trial & A & B & $\mathrm{C}$ & $\mathrm{D}$ & $\mathrm{E}$ & $\mathrm{F}$ & G & $\mathrm{H}$ & Mass loading $(\mu \mathrm{g})$ & Sampling rate (mL/min) \\
\hline 1 & 1 & 1 & 1 & 1 & 1 & 1 & 1 & 1 & $8.20 \times 10^{-3}$ & $1.68 \times 10^{-2}$ \\
\hline 2 & 1 & 1 & 2 & 2 & 2 & 2 & 2 & 2 & $1.32 \times 10^{-2}$ & $9.34 \times 10^{-3}$ \\
\hline 3 & 1 & 1 & 3 & 3 & 3 & 3 & 3 & 3 & $1.41 \times 10^{-2}$ & $5.85 \times 10^{-3}$ \\
\hline 4 & 1 & 2 & 1 & 1 & 2 & 2 & 3 & 3 & $4.14 \times 10^{-2}$ & $7.37 \times 10^{-3}$ \\
\hline 5 & 1 & 2 & 2 & 2 & 3 & 3 & 1 & 1 & $5.40 \times 10^{-3}$ & $5.71 \times 10^{-3}$ \\
\hline 6 & 1 & 2 & 3 & 3 & 1 & 1 & 2 & 2 & $4.48 \times 10^{-2}$ & $1.63 \times 10^{-2}$ \\
\hline 7 & 1 & 3 & 1 & 2 & 1 & 3 & 2 & 3 & $2.96 \times 10^{-2}$ & $5.41 \times 10^{-3}$ \\
\hline 8 & 1 & 3 & 2 & 3 & 2 & 1 & 3 & 1 & $1.35 \times 10^{-1}$ & $1.29 \times 10^{-2}$ \\
\hline 9 & 1 & 3 & 3 & 1 & 3 & 2 & 1 & 2 & $1.45 \times 10^{-2}$ & $8.96 \times 10^{-3}$ \\
\hline 10 & $2(1)$ & 1 & 1 & 3 & 3 & 2 & 2 & 1 & $4.01 \times 10^{-3}\left(1.13 \times 10^{-2}\right)^{\mathrm{a}}$ & $2.50 \times 10^{-3}\left(7.85 \times 10^{-3}\right)$ \\
\hline 11 & $2(1)$ & 1 & 2 & 1 & 1 & 3 & 3 & 2 & $4.91 \times 10^{-3}\left(1.23 \times 10^{-2}\right)$ & $1.99 \times 10^{-3}\left(5.12 \times 10^{-3}\right)$ \\
\hline 12 & $2(1)$ & 1 & 3 & 2 & 2 & 1 & 1 & 3 & $1.49 \times 10^{-3}\left(8.34 \times 10^{-3}\right)$ & $4.00 \times 10^{-3}\left(2.08 \times 10^{-2}\right)$ \\
\hline 13 & $2(1)$ & 2 & 1 & 2 & 3 & 1 & 3 & 2 & $1.22 \times 10^{-2}\left(7.25 \times 10^{-2}\right)$ & $2.21 \times 10^{-3}\left(1.34 \times 10^{-2}\right)$ \\
\hline 14 & $2(1)$ & 2 & 2 & 3 & 1 & 2 & 1 & 3 & $3.04 \times 10^{-3}\left(8.75 \times 10^{-3}\right)$ & $3.87 \times 10^{-3}\left(9.82 \times 10^{-3}\right)$ \\
\hline 15 & $2(1)$ & 2 & 3 & 1 & 2 & 3 & 2 & 1 & $5.89 \times 10^{-3}\left(1.39 \times 10^{-2}\right)$ & $1.93 \times 10^{-3}\left(5.36 \times 10^{-3}\right)$ \\
\hline 16 & $2(1)$ & 3 & 1 & 3 & 2 & 3 & 1 & 2 & $4.78 \times 10^{-3}\left(1.09 \times 10^{-2}\right)$ & $2.62 \times 10^{-3}\left(6.87 \times 10^{-3}\right)$ \\
\hline 17 & $2(1)$ & 3 & 2 & 1 & 3 & 1 & 2 & 3 & $1.62 \times 10^{-2}\left(7.43 \times 10^{-2}\right)$ & $3.02 \times 10^{-3}\left(1.49 \times 10^{-2}\right)$ \\
\hline 18 & $2(1)$ & 3 & 3 & 2 & 1 & 2 & 3 & 1 & $2.15 \times 10^{-2}\left(8.32 \times 10^{-2}\right)$ & $2.08 \times 10^{-3}\left(7.89 \times 10^{-3}\right)$ \\
\hline
\end{tabular}

\footnotetext{
${ }^{a}$ The figures in parentheses indicate results obtained in accordance with the arrangement of experiment variables in column A of the orthogonal array.
} 
Table 3

ANOVA table for identifying significant factors on variance of sampling rates where Car/PDMS SPME fibers were utilized to conduct diffusive sampling for methylene chloride in accordance with orthogonal array design listed in Table 2

\begin{tabular}{|c|c|c|c|c|c|c|c|c|}
\hline \multirow[t]{2}{*}{ Factor } & \multicolumn{3}{|c|}{ Sampling rate $(\mathrm{mL} / \mathrm{min})$} & \multicolumn{5}{|c|}{ Analysis of variance } \\
\hline & Level 1 & Level 2 & Level 3 & d.f. & Mean square & $F$ & Pure sum of square ${ }^{a}$ & Percent influence ${ }^{\mathrm{b}}(\%)$ \\
\hline A & $1.01 \times 10^{-2}$ & - & - & - & - & - & - & \\
\hline $\mathrm{C}$ & $9.65 \times 10^{-3}$ & $9.66 \times 10^{-3}$ & $1.09 \times 10^{-2}$ & 2 & $9.01 \times 10^{-6}$ & 11.2 & $1.64 \times 10^{-5}$ & $1.42^{*}$ \\
\hline $\mathrm{D}$ & $9.78 \times 10^{-3}$ & $1.04 \times 10^{-2}$ & $9.96 \times 10^{-3}$ & 2 & $2.09 \times 10^{-6}$ & 2.58 & $2.56 \times 10^{-6}$ & 0.220 \\
\hline E & $1.03 \times 10^{-2}$ & $1.05 \times 10^{-2}$ & $9.47 \times 10^{-3}$ & 2 & $4.82 \times 10^{-6}$ & 5.97 & $8.03 \times 10^{-6}$ & 0.700 \\
\hline Other/error & & & & 41 & $8.08 \times 10^{-7}$ & & & 3.71 \\
\hline
\end{tabular}

\footnotetext{
* $p<0.05$; significant level $\alpha=0.05$.

a The "pure sum of square" is the value that the "sum of the square" minus "the product of the degree of freedom (d.f.) and the error variance".

b The "percent influence" is the percentage where the "pure sum of square" is divided by the "total sum of square".
}

Temperatures of 15,25 , and $30^{\circ} \mathrm{C}$, and relative humidities of 0 , 50 , and $80 \%$ were set because most ambient air measurements approach these ranges. Toluene was chosen to demonstrate interference in the competitive adsorption study, because toluene and methylene chloride are the main ingredients in paint-stripping products [16]. The path length was set at 3,10 , and $15 \mathrm{~mm}$, that is within the adjustable range $(3-30 \mathrm{~mm})$ of the SPME needle. The sampling time were set at 5, 15 and 30 min that include the short-term exposure level (STEL) allowing to perform a $15 \mathrm{~min}$ TWA sampling [17].

The mass loading and the sampling rate were considered to be the experimental outcomes (Table 2). The average effect of a factor at a level was calculated by examining the orthogonal array, the factor assignment and the experimental outcomes. All outcomes of a factor at the same level were averaged (Table 3 ). The analysis of variance (ANOVA) was performed to identify the influence of individual factor to variance of outcomes. The percent influence of a factor was calculated by the "pure" sum of squares over a "total" sum of squares [18].

\subsection{Materials}

All SPME fibers and holders were purchased from Supelco (Bellefonte, PA). Methylene chloride (99.5\%), toluene (99.9\%) and $n$-hexane $(99.5 \%)$ were procured from Merck (Darmstadt, Germany). Gas-tight syringes and microsyringes for sample preparation and gaseous injection were purchased from Hamilton (Reno, NV).

\subsection{Instrumentation}

All analyses were performed on a HP-5890 series II (Agilent Tech., Palo Alto, CA) gas chromatograph equipped with an electron capture detector. The column was a $30 \mathrm{~m} \times 0.53 \mathrm{~mm}$ I.D. 0.1- $\mu \mathrm{m}$ film DB-wax chemically bounded fused-silica capillary column (J\&W Scientific, Folsom, CA). The carrier gas was nitrogen with a flow rate of $0.3 \mathrm{~mL} \mathrm{~min}^{-1}$ for $1 \mathrm{~min}$, which was shifted to $2 \mathrm{~mL} \mathrm{~min}^{-1}$ in a splitless-injection mode. The temperature for the injector port was $180^{\circ} \mathrm{C}$. The oven temper- ature was isothermal at $70^{\circ} \mathrm{C}$ for $8 \mathrm{~min}$. The analyte of SPME sampling was thermally desorbed from the fiber in the injector port at temperature of $180^{\circ} \mathrm{C}$.

\subsection{Sampling and analysis}

\subsubsection{Theory}

The SPME assembly can be used as a diffusive sampler, simply by retracting the sorbent coating fiber into the needle housing a known distance $Z$ from the opening of fixed area $A$ (Fig. 1) [10]. According to Fick's first law, the sampling rate of a chemical using the sampler can be defined as follows:

$\mathrm{SR}=D \times\left(\frac{A}{Z}\right)$

where $D$ is the diffusion coefficient of the chemical in gas phase. The diffusion coefficient of methylene chloride in the atmosphere can be estimated using Gilliland's approximation, of $0.1037 \mathrm{~cm}^{2} \mathrm{~s}^{-1}$, at $25^{\circ} \mathrm{C}$ and $760 \mathrm{mmHg}$. With the opening of

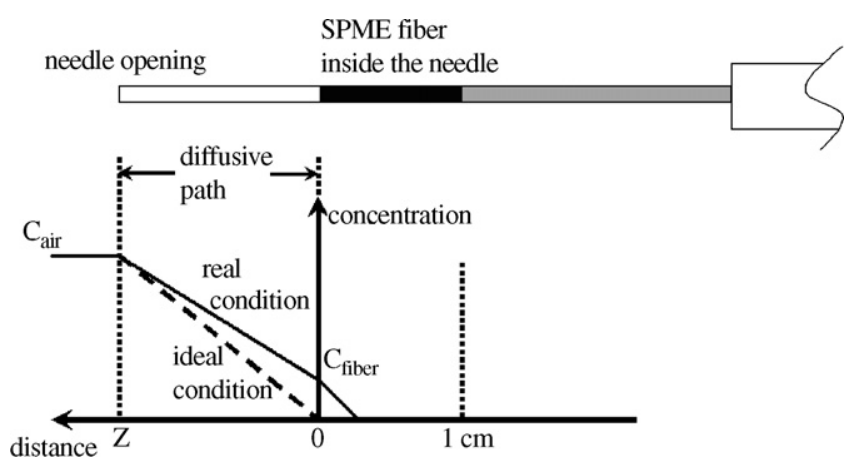

Fig. 1. Schematic diagram depicts a SPME assembly where a SPME fiber is retracted and housed in a needle for diffusive sampling. In diffusive sampling, $C_{\text {air }}$ designates concentration of an analyte in ambient air; $Z$ is the path length between the opening of a needle and the SPME fiber; the opening of the crosssectional area is denoted as $A$; the length of a SPME fiber rod used in this study is $1 \mathrm{~cm}$; concentration of an analyte on the surface of a fiber is supposedly to be zero in the initial stage of sampling and then build up to $C_{\text {fiber }}$ as sampling proceeds for a while. Thus, real sampling efficiency would deviate from the prediction based on Fick's first law. 


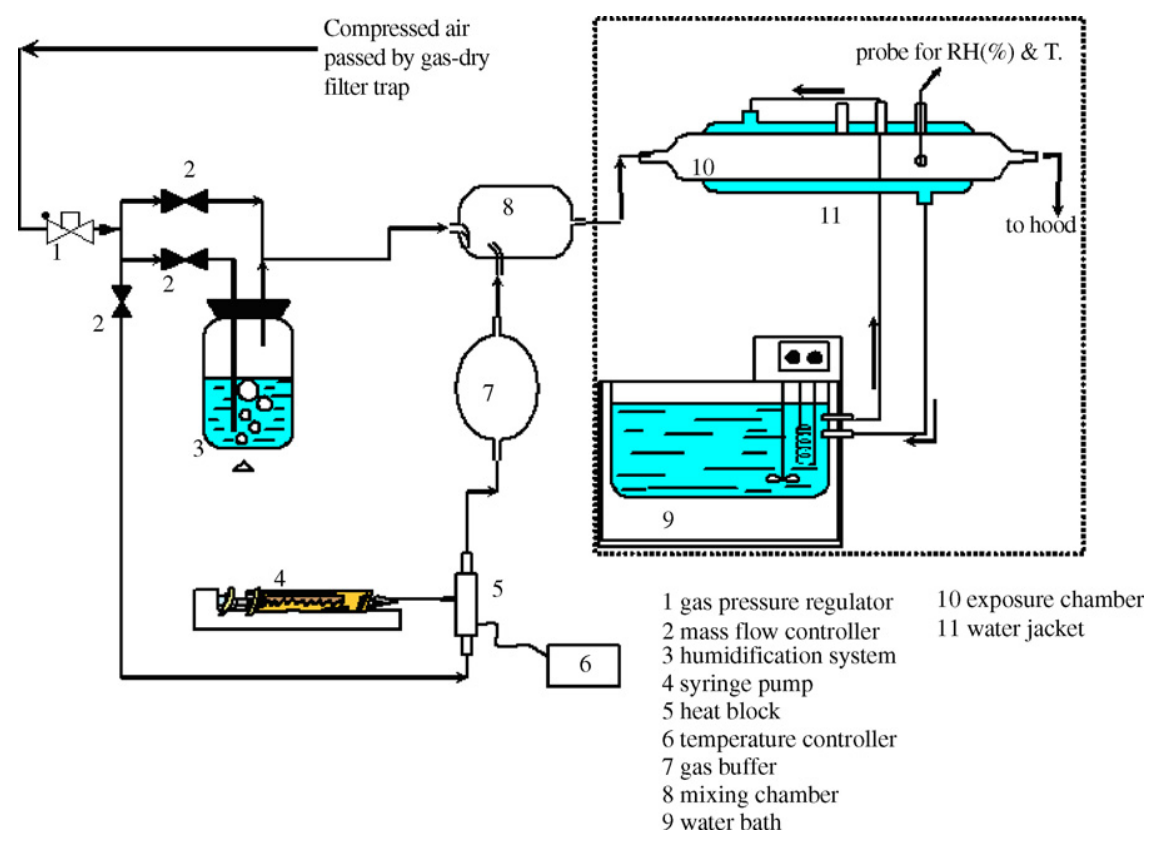

Fig. 2. Schematic diagram of the system for generating known concentration of methylene chloride.

the needle area being $0.00086 \mathrm{~cm}^{2}$ and the diffusive path set at 3 , 10 and $15 \mathrm{~mm}$, the sampling rate for methylene chloride was estimated to be $1.8 \times 10^{-2}, 5.4 \times 10^{-3}$ and $3.6 \times 10^{-3} \mathrm{~mL} \mathrm{~min}^{-1}$, respectively.

\subsubsection{Preparation of reference vapor of methylene chloride}

Known concentrations of methylene chloride and a mixture of methylene chloride and toluene were prepared by the dynamic standard gas generator as shown in Fig. 2. The methylene chloride or the mixture in a microsyringe was injected at a constant rate regulated by a syringe pump (Sage Instruments, Boston, MA), and evaporated by a heat block (Km-2011, Kung-Ming, Taiwan) at a temperature of $200{ }^{\circ} \mathrm{C}$. Compressed air through a gas-dry filter trap (Model 75-10, Balston, Haverhill, MA) as a dilution gas stream merged with the chemical-containing air stream and a humidity-conditioning air stream. The combined air stream was then led into a water-jacket exposure chamber where temperature was regulated by a temperature-controlled water bath (Chin-Hsin Enterprise Co., Taipei, Taiwan). The flow rates in this system were regulated by mass flow controllers (Porter Instruments, Hatfield, PA) and calibrated by a bubble generator (Sensidyne Inc., Clearwater, FL). The temperature and relative humidity were measured by a thermo-hydrometer from Testo (Lenzkirch, Germany).

The experimental trials of sampling were performed in the exposure chamber while after an aliquot of $200 \mu \mathrm{L}$ of gas was drawn from the chamber and had it injected directly into GC for double-check the concentration of methylene chloride.

\subsubsection{Preparation of methylene chloride calibration curve}

The air bag method was used to prepare the known concentrations $(0,50$ and $100 \mathrm{ppm})$ of methylene chloride gas. An appropriate aliquot of methylene chloride was injected into $1 \mathrm{~L}$ sample bags (SKC Inc., Fullerton, CA) containing $800 \mathrm{~mL}$ of purified air. An aliquot (10-250 $\mu \mathrm{L})$ of the prepared gas was drawn by a gas-tight microsyringe and injected directly into GC. The calibration curve was plotted by response versus mass of methylene chloride injected. The relative errors of using the calibration curve for prediction were controlled within $10 \%$.

\section{Results and discussion}

\subsection{Loading capacity of different SPME fibers}

Table 2 lists the outcomes of the mass loading and the sampling rate upon the 18 trials that were designed on the basis of Taguchi's orthogonal array. The average mass loading of methylene chloride on Car/PDMS fibers in nine trials (numbered 1-9) was $3.41 \times 10^{-2} \mu \mathrm{g}$ while the average mass loading on polyacrylate fibers in nine trials (numbered 10-18) was $8.23 \times 10^{-3} \mu \mathrm{g}$. The pooled coefficients of variance between trials were $5.01 \%$ for Car/PDMS and $5.70 \%$ for polyacrylate. The Car/PDMS fibers obviously have better loading capability for sampling methylene chloride than polyacrylate fibers have.

\subsection{Sampling rates}

To determinate the sampling rates of using different fibers, a plot of the collected mass versus the product of concentration and exposure time was performed (Fig. 3). The sampling rates of using Car/PDMS were $1.4 \times 10^{-2}, 7.7 \times 10^{-3}$ and $5.1 \times 10^{-3} \mathrm{~mL} \mathrm{~min}^{-1}$ as the diffusive path lengths were set at 3 , 10 , and $15 \mathrm{~mm}$, respectively (Fig. 3A), while the sampling rates of using polyacrylate fiber were $2.3 \times 10^{-3}, 1.9 \times 10^{-3}$ and $5.0 \times 10^{-4} \mathrm{~mL} \mathrm{~min}^{-1}$ (Fig. 3B). The type of adsorbent apparently affected the sampling rate. However, they differed from 

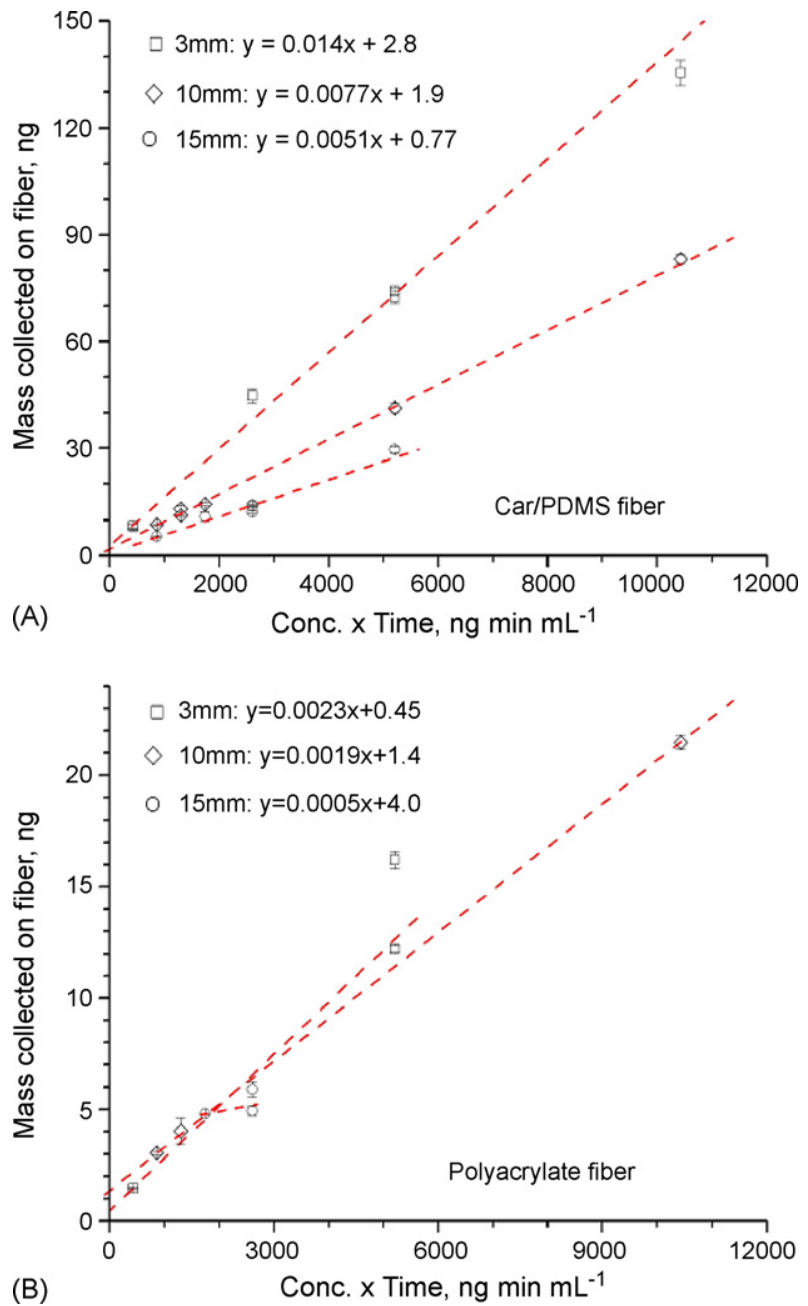

Fig. 3. The relationship between the measured mass and the product of concentration and sampling time as the SPME fibers at three diffusive path length settings (3,10 and $15 \mathrm{~mm}$ ) were exposed to methylene chloride at concentration of 1/2 TLV, 1 TLV (50 ppm) and 2 TLV ((A) for Car/PDMS fiber, $n=18$; (B) for polyacrylate fiber, $n=9$; slope indicates the sampling rate in $\mathrm{mL} \mathrm{min}^{-1}$ ).

those predicted by Fick's first law (Table 4). The sampling rate of using Car/PDMS with diffusive path of 10 or $15 \mathrm{~mm}$ was greater than theoretical value but it was smaller than theoretical value as using Car/PDMS with path length of $3 \mathrm{~mm}$. The results in this study seem not completely agree to Hafkenscheid's proposal that the effective sampling rate of a diffusive sampler $\left(U_{\text {eff }}\right)$

Table 4

Comparison of the measured sampling rates to the theoretical values predicted by Fick's first law

\begin{tabular}{llll}
\hline $\begin{array}{l}\text { Diffusive path } \\
\text { length }(\mathrm{mm})\end{array}$ & \multicolumn{2}{l}{ Sampling rate $\left(\mathrm{mL}^{-1} \min \right)$} & $e^{\mathrm{a}}$ \\
\cline { 2 - 3 } & Theoretical & Measured & \\
\hline 3 & $1.78 \times 10^{-2}$ & $1.36 \times 10^{-2}$ & 0.76 \\
10 & $5.40 \times 10^{-3}$ & $7.67 \times 10^{-3}$ & 1.42 \\
15 & $3.60 \times 10^{-3}$ & $5.11 \times 10^{-3}$ & 1.42 \\
\hline
\end{tabular}

a The sampling efficiency " $e$ " is a ratio of a measured sampling rate over a theoretical sampling rate.

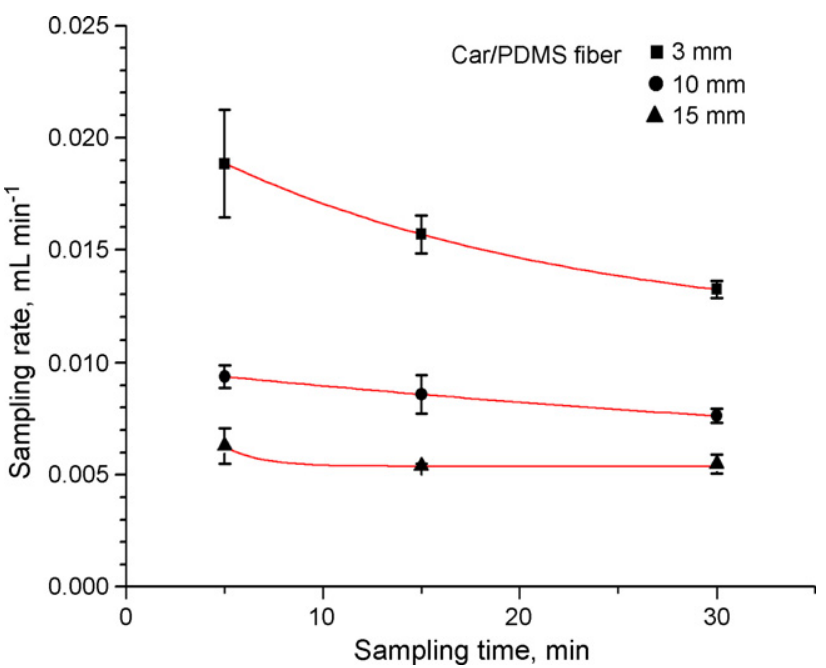

Fig. 4. The Sampling rate of methylene chloride by Car/PDMS fiber at three different diffusive path settings $(3,10$ and $15 \mathrm{~mm})$ varied with increment of sampling time.

would be [20]:

$U_{\text {eff }}=D\left(\frac{A}{Z}\right) \times e$

where " $e$ " is defined as the sampling efficiency and $0<e<1$. The greater sampling rates as demonstrated in this study are likely due to the adsorption of methylene chloride on the stainless steel needle, similar as the results of Chen and Pawliszyn's work in that sampling rate obtained with the stainless steel needle fiber were significantly higher than the theoretical values for the first 2-h sampling for $n$-undecane, while those determined by the deactivated needle fiber were consistent with theoretical values and were highly reproducible [19].

Fig. 4 depicts that sampling rates varied with sampling time as well as the length of diffusion path. The sampling rate decreased slightly with increase of sampling time until they leveled off, especially in the trials when the diffusion path was set at 3$\mathrm{mm}$. Sampling rates determined by the Car/PDMS fiber with the diffusion path of 3-mm were significantly higher than the theoretical value $\left(1.78 \times 10^{-2} \mathrm{~mL} \mathrm{~min}^{-1}\right.$ in Table 4$)$ for the first 5 min sampling. This result also resembles the observation of a typical tube-type diffusive sampler in which the initial sampling rate was close to the estimation by Fick's first law, but decreased gradually as the sampling continued [21]. Fick's first law describes the mass transfer of the analyte in the air phase. In the air phase, only diffusion was taken into account and the sorbent of a diffusive sampler should be thought as a 'zero sink' for the target analytes. Although a sorbent would be an ideal 'zero sink' at the every beginning for a diffusive sampling, analyte on the surface of an adsorbent would build up and migrates into the adsorbent as the sampling proceeds. The sampling rate will be constant only after analyte reaches phase equilibrium. The time required to reach a constant sampling rate was dependent on the length of the diffusive path and concentration of analyte. The shorter the diffusive path, the shorter the time required. But, the results from the trials of using samplers with path length of 10 and $15 \mathrm{~mm}$ (Fig. 4) did not obviously 
show up the expectation just as Chen and Pawliszyn demonstrated in their study [19], probably because the sampling times in the trials were within $30 \mathrm{~min}$ that would be not long enough for demonstration. However, our results imply that, for assessing short-term exposure level in industrial hygiene purposes, sampling of methylene chloride of concentration around the 0.5 TLV-2.0 TLV by $85 \mu \mathrm{m}$-Car/PDMS fibers, it is better to have the path length set at 10 or $15 \mathrm{~mm}$ because the sampling rates at these path length were almost constant.

\subsection{Influence of environmental factor}

Based on the results in Table 2, sampling rates of a factor at the same level were averaged and ANOVA was employed (Table 3). In Table 3, the influence of diffusive path length on sampling rate accounted for $86.4 \%$ of total variance. Sampling time accounted for $5.73 \%$. The other four factors (i.e., concentration, temperature, relative humidity and the extent of coexistent toluene) accounted for $1.84,1.42,0.70$ and $0.22 \%$, respectively.

Sampling rate was significantly affected by temperature from 15 to $30^{\circ} \mathrm{C}$ in this study $(F=11.2, p<0.05)$. Diffusivity of an analyte is a function of temperature and pressure and alters by factors of $0.5 \% / \mathrm{K}$ and $0.1 \% / \mathrm{mbar}$ according to Gilliland's approximation. Relative humidity was not statistically significant factor $(F=2.58, p>0.05)$. It seems that relative humidity $\leq 80 \%$ did not significantly interfere the diffusive sampling of methylene chloride with a Car/PDMS SPME fiber. This is similar to the result obtained by a $100 \mu \mathrm{m}$ of PDMS fiber when the hydrophilic fiber was exposed to relative humidity below $90 \%$ [22].

\subsection{Analysis and quality of control}

In order to check the reference concentration of methylene chloride, $200-\mu \mathrm{L}$ of chamber gases were drawn and directly

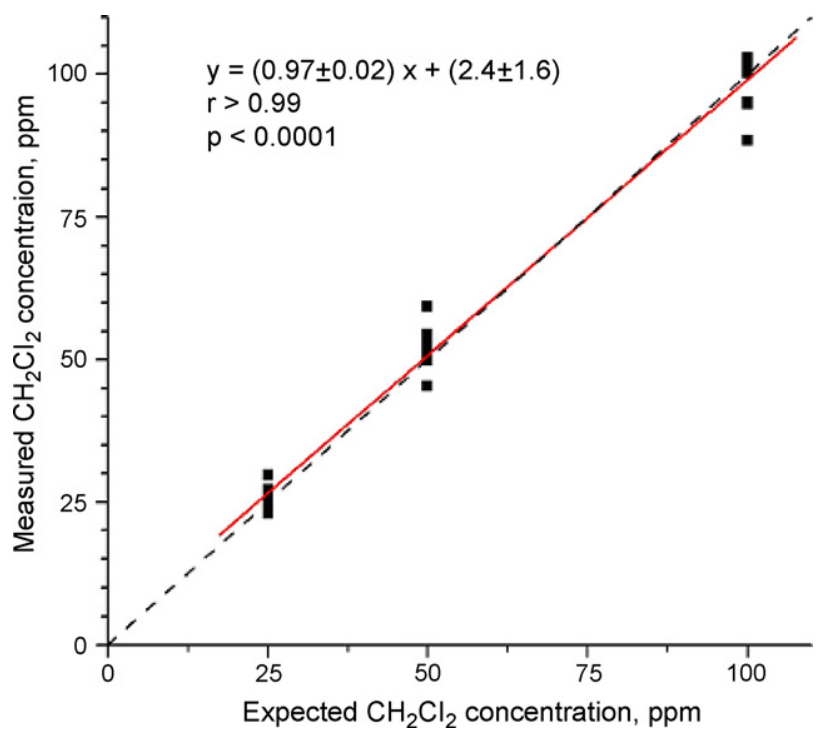

Fig. 5. The measured concentration determined by pick-up with micro-syringe and analysis with GC/ECD vs. the expected concentration in the exposure chamber of the system for generating gaseous methylene chloride $(n=27)$.

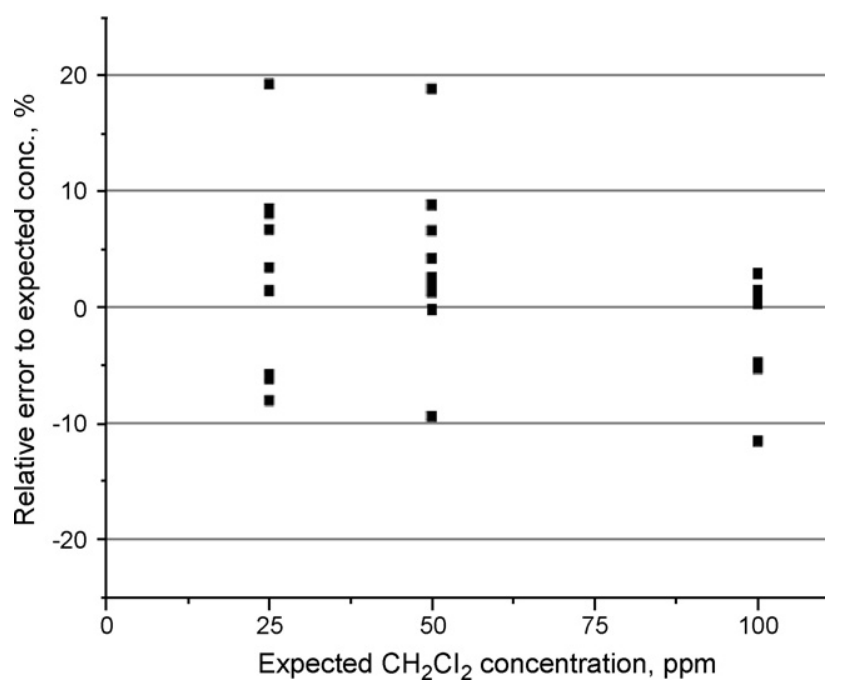

Fig. 6. The relative errors of the measured concentration vs. the expected concentration in the exposure chamber of the system for generating gaseous methylene chloride $(n=27)$.

injected into the GC/ECD for analysis. As shown in Fig. 5, the observed concentration was well correlated with the expected value $(r>0.99, p<0.0001, n=27)$. Of the 27 analyses, $89 \%$ evidenced an error of less than $10 \%$ relative to the expected concentration (Fig. 6).

Fig. 7 shows gas chromatograms of methylene chloride detected by GC/ECD. The retention time of methylene chloride was $5.6 \mathrm{~min}$. The peak corresponding to toluene does not show up because its retention time is 8.6 min that is longer than the running time showing in the figure. During preliminary test, it was also noticed that the peak response to a $200-\mu \mathrm{L}$ gaseous

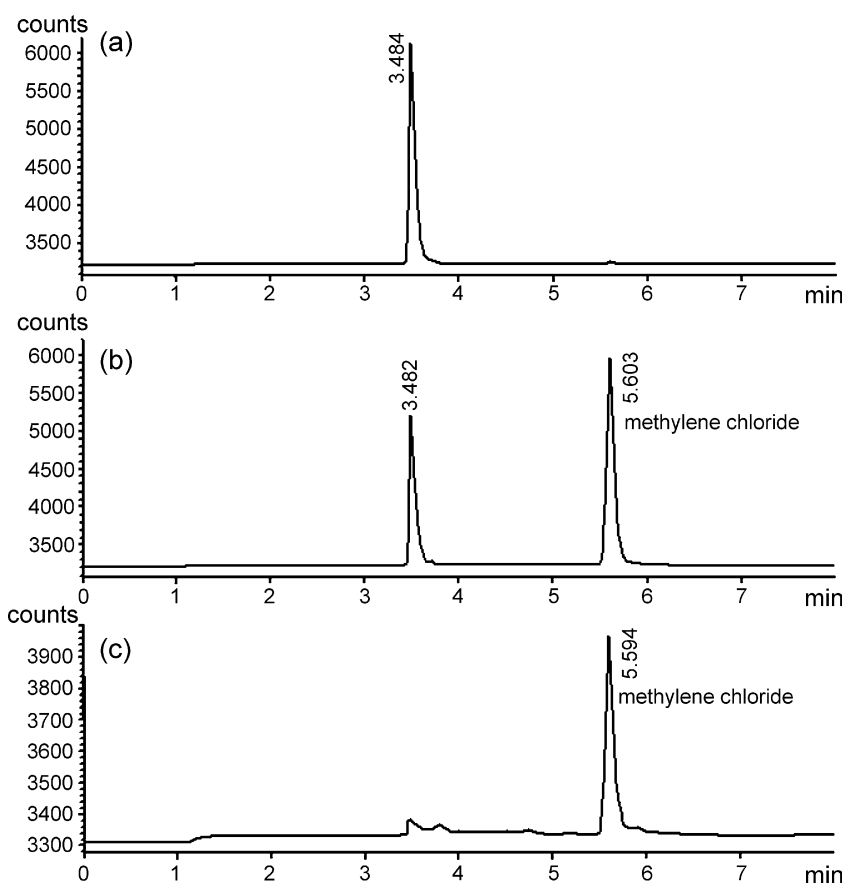

Fig. 7. The GC/ECD chromatograms of methylene chloride ((a) air-blank; (b) $100 \mu \mathrm{L}$ gaseous methylene chloride of $50 \mathrm{ppm}$ by direct injection; (c) sampling of $50 \mathrm{ppm}$ methylene chloride for $5 \mathrm{~min}$ by Car/PDMS fiber). 
injection of $100 \mathrm{ppm}$ toluene was hard to detect. Thus, the peak about toluene was neglected in the latter runs. The ECD is relatively sensitive to halogen-containing compounds so that the coexistent nonhalogen-containing compound like toluene would make less interference on a chromatogram.

\section{Conclusion}

Methylene chloride is one of possible carcinogens that are recommended to low down exposure level. The SPME sampling assembly could be an effective device for evaluating this hazard. In this study, the SPME diffusive sampling was set for assessing the STEL of methylene chloride in industrial scenarios. Taguchi's orthogonal array was utilized to design the experimental trials and ANOVA was performed to identify the main concerns on the performance of SPME diffusive sampling. The use of orthogonal array helps us study many factors simultaneously and reduce the number of experiment to a much practical and affordable size. The results elucidate that the sampling rates of a SPME fiber assembly varied with sampling time once the length of the diffusive path was fixed. The measured sampling rates were greater than the theoretical values, and decreased with increment of sampling time until they came to constant. To evaluate the STEL in industrial scenario for sampling methylene chloride of concentration around 0.5 TLV-2.0 TLV, we recommend using the SPME assembly of $85 \mu \mathrm{m}$-Car/PDMS fiber and of path length at 10 or $15 \mathrm{~mm}$.

\section{Acknowledgements}

This study was supported by the Institute of Occupational Safety and Health (IOSH), Council of Labor Affairs, Executive Yuan, Taiwan.

\section{References}

[1] J.D. Wilson, Toxicological Profile for Methylene Chloride: Agency for Toxic Substances and Disease Registry, 2000.

[2] N.Y. Kim, S.W. Park, J.K. Suh, J. Forensic Sci. 41 (1996) 527.

[3] ACGIH, Documentation of the Threshold Limit Values and Biological Exposure Indices: Dichloromethane, American Conference of Governmental Industrial Hygienists, OH, 2001, p. 1.

[4] S. Ghittori, P. Marraccini, G. Franco, Am. Ind. Hyg. Assoc. J. 54 (1) (1993) 27.

[5] C.F. Estill, D.S. Watkins, S.A. Shulman, R.W. Kurimo, R.J. Kovein, AIHA J. 63 (2002) 326.

[6] H. Xiao, S.P. Levine, J. Nowak, AIHA J. 54 (9) (1993) 545.

[7] NMAM, Methylene Chloride-method no. 1005, National Institute of Occupational Safety and Health, Cincinnati, OH, 1994.

[8] J.E. Woodrow, M.M. McChesney, J.N. Seiber, Anal. Chem. 60 (1988) 509.

[9] A. Khaled, J. Pawliszyn, J. Chromatogr. A 892 (2000) 455.

[10] P.A. Martos, J. Pawliszyn, Anal. Chem. 71 (1999) 1513.

[11] S.W. Tsai, S.T. Tsai, V.S. Wang, J.S. Lai, J. Chromatogr. A 1026 (2004) 25.

[12] S.W. Tsai, T.A. Chang, J. Chromatogr. A 954 (2002) 191.

[13] E.R. Gilliland, Ind. Eng. Chem. 26 (1934) 681.

[14] J. Pawliszyn, Solid Phase Microextraction: Theory and Practice, WileyVCH, Inc., New York, NY, 1997, p. 98.

[15] G. Taguchi, The System of Experimental Design: Engineering Methods to Optimize Quality and Minimize Costs, Kraus International, New York, 1987.

[16] J.O. Stull, R.W. Thomas, L.E. James, AIHA J. 63 (2002) 62.

[17] American Conference of Governmental Industrial Hygienists (ACGIH), Threshold Limit Values for Chemical Substances and Physical Agents and Biological Exposure Indices, Cincinnati, OH, 2006.

[18] R.K. Roy, Design of Experiments Using the Taguchi Approach: 16 Steps to Product and Process Improvement, John Wiley \& Sons, Inc., New York, 2001, p. 211.

[19] Y. Chen, J. Pawliszyn, Anal. Chem. 75 (2003) 2004.

[20] T. Hafkenscheid, International Conference Measuring Air Pollutants by Diffusive Sampling, European Commission, Montpellier, France, 2001.

[21] B. Tolnai, A. Gelencser, J. Hlavay, Talanta 54 (2001) 703.

[22] P.A. Martos, J. Pawliszyn, Anal. Chem. 69 (1997) 206. 